\title{
Recombinant Porcine Rotavirus VP6 Proteins Expressed in Lactobacillus plantarum NC8 Strain Induces Specific Mucosal and Systemic Antibody Production in Experimental BALB/c mice
}

SERIA masole SHONYELA ( $\nabla$ seria.masole@sacids.org )

Jilin Agricultural University https://orcid.org/0000-0002-3347-2309

Wentao Yang

Jilin Agricultural University

Guilian Yang

Jilin Agricultural University

Chunfeng Wang

Jilin Agricultural University

Research article

Keywords: Porcine Rotavirus, VP6, Lactobacillus plantarum NC8, immunogenicity, Pigs

Posted Date: January 17th, 2020

DOl: https://doi.org/10.21203/rs.2.21079/v1

License: (c) (i) This work is licensed under a Creative Commons Attribution 4.0 International License.

Read Full License 


\section{Abstract}

\section{Background}

Rotaviruses are the main cause of animal and infant diarrhea and are widely distributed worldwide. In the pig industry, porcine rotavirus infection is a significant cause of mortality and morbidity; therefore, the optimization and well-organized distribution of vaccines for infection prevention is needed. Because immune responses related to protection are mainly mucosal in nature, the induction of mucosal immunity is significant for preventing porcine rotavirus infection.

\section{Methods}

The major protective VP6 antigens against porcine rotavirus (PRV) expressed by Lactobacillus plantarum NC8-pSIP409-pgsA-VP6-Dcpep were used to orally immunize mice. Western blot analysis and SDS-PAGE were used to confirm the expression of recombinant NC8-pSIP409-pgsA-VP6-Dcpep, and immunofluorescence was also used to verify its surface expression on L. plantarum NC8.

Results

High levels of mucosal $\lg A$ and serum immunoglobulin $\mathrm{G}(\mathrm{IgG})$ were produced in mice that were orally immunized with recombinant $L$. plantarum NC8-pSIP409-pgsA-VP6-Dcpep. Mice immunized with NC8pSIP409-pgsA-VP6-Dcpep had higher IgA titers than mice immunized with NC8-pSIP409-pgsA and PBS. The mice orally immunized with NC8-pSIP409-pgsA-VP6-Dcpep demonstrated a virus challenge-induced mean fever peak of $38.6^{\circ} \mathrm{C}$ in the NC8-pSIP409-pgsA-VP6-Dcpep group and $41.1^{\circ} \mathrm{C}$ in the NC8-pSIP409pgsA group 4 to 6 days after the challenge, and 7 to 9 days following challenge.

\section{Conclusion}

Our results confirmed that with regard to stimulating mucosal immunity, the VP6 genes of the porcine rotavirus (PRV) Chinese PRV isolate DN30209 strain expressed by L. plantarum NC8 is effective and that Dcpep is superior in its ability to stimulate mucosal immunity, suggesting that this approach can be implanted in pigs.

\section{Background}

Rotaviruses are members of the family Reoviridae. Depending on their particular inner capsid protein sequences, porcine rotaviruses are categorized into groups A, B and C [1]. Rotaviruses have a nonenveloped, double-stranded RNA genome that is composed of 11 segments enclosed by a triplelayered icosahedral capsid, [2-5]. The main source of acute diarrhea in piglets is porcine rotaviruses, which can cause high rates of mortality and morbidity [6-11]. In both pre- and post-weaning pigs, rotavirus Group A is the main source of rotavirus-associated diarrhea and accounts for $54 \%$ and $45 \%$ of the diarrhea experienced in those populations of pigs, respectively [1]. Some research has reported that in commercial pig farms, $89 \%$ of all rotavirus-associated diarrhea can be attributed to group A rotavirus 
infections [12]. Rotavirus outbreaks are difficult to prevent because they are transmitted via the fecal oral route and can survive in the environment for a long period of time. Replication of the virion takes place in the intestinal villi in epithelial cells and destroys enterocytes mainly in the ileum and jejunum, leading to villous atrophy [13-16]. In addition, in the affected regions, nutrients cannot be absorbed or digested, causing severe malabsorption [13-16]. The control of rotavirus infections requires a solid understanding of the epidemiology of rotaviruses, which will contribute to prevention programs and improve the current vaccines. Presently, the obtainable vaccines cannot provide adequate protection. To activate immunocompetence, repeated administrations and a large vaccine dose are generally needed. This repeatedly causes unwanted clinical signs. To overcome these weaknesses and deliver antigens to the mucosal immune system, possible improvements to lactic acid bacteria (LAB) have been proposed.

Mucosal immunity plays an important role in protective immunity because rotaviruses are enteric pathogens. In the gut, innate immune responses initiate acquired immune responses and offer the first line of protection against pathogenic microorganisms. In addition, the only appropriate way of eliciting gut immunity is to prompt the immune response via oral immunization because this oral route assists in gut-associated lymphoid tissue (GALT) stimulation, improving anti-viral IgA production $[17,18]$.

Live vaccines stimulate the most efficient defensive responses, unlike heat-killed or recombinant antigen formulations, because they elicit both mucosal and systemic immunity $[17,18]$. Repeated vaccinations and large vaccination doses are needed due to the challenges posed by oral vaccination, such as the fact that the gut environment habitually deactivates and/or denatures potential vaccinogens, causing fever and diarrhea, with the live vaccine often being shed in the feces $[17,18]$. With regard to the stimulation of mucosal immunity, lactic acid bacteria (LAB) can be used to overcome these challenges [19].

Furthermore, several LAB strains are capable of colonizing and surviving the intestinal tract, stimulating nonspecific immunoadjuvant consequences [19]. It is essential to boost the immunogenicity of genetically engineered vaccines by combining them with suitable adjuvants because they are poorly immunogenic and composed of a single recombinant antigen. The dendritic cells play a central role in targeting peptide by directing innateand regulating adaptive/acquired immunity. By phage display the three DC-binding 12 number of residues in the peptide 12-mer: pep3 FYPSYHSTPQRP, pep12 AYYKTASLAPAE and pep18SLSLLTMPGNAS was recognized in 2004. Out of the three peptide pep3 later was renamed Dcpep, for the reason that Dcpep bound to both immature and mature DCs in a saturable way, than pep12 or pep18 and had the fascinating property to bind both human Monocyte-Derived Dendritic CellsmdDCs and mouse CD11C+ I-A+ DCs [20]

Herein, we constructed recombinant L. plantarum NC8 strains expressing porcine rotavirus VP6 to test the effectiveness of the expression of the VP6 porcine rotavirus protein by L. plantarum for oral vaccinations and the performance of $L$. plantarum as an antigen delivery system. Following the administration of live bacteria to mice, the immunogenic ability of these recombinant strains was analyzed. The oral administration of the recombinant strain NC8-pSIP409-pgsA-VP6-Dcpep stimulated specific anti-rotavirus systemic and mucosal immune responses. In mice immunized with L. plantarum NC8 expressing the VP6-Dcpep fusion, the effectiveness of the immune response evaluation was greater than in mice 
immunized with NC8-pSIP409-pgsA, indicating the effectiveness of Dcpep as a mucosal adjuvant. Using standard molecular biology techniques, the recombinant NC8-pSIP409-pgsA-VP6-Dcpep was constructed to recognize DCs and the VP6-antigen more efficiently in the intestinal mucosa, and its immunogenicity was further investigated. The results indicated that the in vivo antibody levels were substantially improved after BALB/c mice were immunized by the lavage administration of our recombinant NC8pSIP409-pgsA-VP6-Dcpep.

\section{Results}

\section{Cloning, expression and purification of Rotaviral target genes VP6 in Prokaryotic Expression System}

The recombinant plasmids were identified by restriction endonuclease digestion enzyme Xba I and Hind III digestion. As shown in Fig. 1, two distinct bands appeared which were in accordance with the expected sizes. The results of sequencing were compared by NCBI Blast, and the homology was $100 \%$.

\section{Flow cytometry detection of surface fluorescence intensity of L. plantaram NC8-pSIP409-pgsA-VP6-Dcpep.}

To confirm VP6 expression, the recombinant NC8-pSIP409-pgsA-VP6-Dcpep was SppIP-induced and cultured. Anti-VP6 serum from rabbits was used as an antibody; IgG from goats labeled with FITC was used as the secondary fluorescent antibody. FlowJo 7.6.1 software was used for detection. The results showed that the peak value of NC8-pSIP409-pgsA-VP6-Dcpep group was 92.7\%, compared with $57.3 \%$ count in the NC8-pSIP409-pgsA group, as shown in Fig. 2. The expression of VP6 protein on the surface of $L$. plantarum could be inferred from the obvious right shift.

\section{Expression of VP6-DCpep in L. plantarum NC8}

NC8-pSIP409-pgsA-VP6-Dcpep supplemented with SPPIP was cultured in basal MRS medium. The SPPIP-induced NC8-pSIP409-pgsA-VP6-Dcpep was subjected to SDS-PAGE, and the corresponding VP6 recombinant proteins were confirmed at $48 \mathrm{kDa}$ by Coomassie blue staining analysis (Fig 3 SDS-PAGE lanes 1-3). Then, the anti-VP6 antibodies were observed by Western blot analysis by running the gels in parallel and transferring them to nitrocellulose membranes. The results are shown in Fig 3 , in the Western blot lanes 3 and 4 . The SDS-PAGE results showed that there was a clear band at $48 \mathrm{kDa}$. The Western blot results showed a clear protein band at $48 \mathrm{kDa}$, which was consistent with the SDS-PAGE results. As a negative control, NC8-pSIP409 had no imprinting band after Western blot analysis, as shown in Figure 3 (lane 1). These results indicated that VP6 protein was expressed on the cell wall surface of L. plantarum NC8, which was reactive. 


\section{Immunofluorescence analysis}

VP6 was detected on the surface of induced cells cultured with NC8-pSIP409-pgsA-VP6-Dcpep (Fig 4). No immunofluorescence was observed for non-induced NC8-pSIP409-pgsA, although it was incubated in a similar manner (Fig 4 NC8-pSIP409-pgsA). Immunofluorescence analysis of SppIP-induced L. plantaram NC8 indicated that there were green-yellow fluorescence reactions on the surface of SPPIP-induced recombinant NC8-pSIP409-pgsA-VP6-Dcpep.

\section{Antibody responses following oral immunization}

To assess the capability of the recombinant L. plantaram NC8-pSIP409-pgsA-VP6-Dcpep to induce systemic and mucosal immunity, the presence of anti-VP6 IgG and IgA antibodies was determined. After immunization, the serum, feces and bronchoalveolar lavage fluid of the mice were collected, and the expression levels of specific $\lg G$ and slgA were detected by indirect ELISA. The results are shown in Fig 5 A. Compared with the empty vector group, the NC8-pSIP409-pgsA-VP6-Dcpep group had significantly increased serum IgG content $(P<0.01)$. The NC8-pSIP409-pgsA-VP6-Dcpep group feces and bronchoalveolar lavage fluid slgA content was significantly increased compared with that of the empty vector group $(P<0.05, P<0.01)$. There were no significant differences in the NC8-pSIP409-pgsA-VP6-Dcpep group, and the results are shown in Fig 5 B. The above results indicate that the NC8-pSIP409-pgsA-VP6Dcpep group had significantly increased expression of specific $\lg G$ and $\operatorname{sig} A$ and had good anti-microbial infection ability.

\section{Effect of NC8-pSIP409-pgsA-VP6-Dcpep on Specific Antibody in Mice}

To further evaluate the role of NC8-pSIP409-pgsA-VP6-Dcpep in humoral immunity, we used an ELISA kit to detect the levels of the cytokines IFN-gamma and IL-4 in the serum. The results showed that compared with the empty carrier group, the content of IL-4 in the NC8-pSIP409-pgsA-VP6-Dcpep group was significantly greater $(P<0.001)$. There was also significant difference $(P<0.001)$ in IFN-gamma between the NC8-pSIP409-pgsA-VP6-Dcpep group and the blank carrier group (Fig 6).

\section{Effect of NC8-pSIP409-pgsA-VP6-Dcpep on Mouse B Cells}

To further evaluate the effect of NC8-pSIP409-pgsA-VP6-Dcpep on mouse B cells, we used flow cytometry to detect the expression of intracellular IgA in B cells of mouse PP, as shown in Fig 7, compared with the empty vector group, the NC8-pSIP409-pgsA-VP6-Dcpep group. The expression of B220+lgA+ B cells in the PP node was significantly increased $(P<0.05)$ 


\section{Vaccinated mice against PRV challenge}

To assess the protective effect of NC8-pSIP409-pgsA-VP6-Dcpep, a viral challenge experiment was performed in mice against PRV infection postvaccination, we infected mice with rotavirus after second booster immunization. As shown in Figures 8, NC8-pSIP409-pgsA-VP6-Dcpep groups were able to produce immunity in mice. The results demonstrated that an effectual defense rate of $81 \%$ against PRV challenge was detected in mice immunized with the NC8-pSIP409-pgsA-VP6-Dcpep, which was higher than the $59.2 \%$ in the mice immunized with NC8-pSIP409-pgsA; the control group of mice that received only PBS displayed severe clinical symptoms of infection after the viral challenge (Fig 8). The mice orally immunized with NC8-pSIP409-pgsA-VP6-Dcpep demonstrated a virus challenge-induced mean fever peak of $38.6^{\circ} \mathrm{C}$ (result not shown) in the NC8-pSIP409-pgsA-VP6-Dcpep group and $41.1^{\circ} \mathrm{C}$ in the NC8-pSIP409pgsA group 2 to 5 days after the challenge, and 6 to 9 days following challenge, all mice were survived and recovered gradually from the clinical signs of PRV infection. A longer fever period with a mean fever peak of $42.1^{\circ} \mathrm{C}$ (result not shown) was observed in the PBS group of mice following viral infection until euthanasia (Fig 8).

Because the lactic acid bacteria expressing rotavirus mainly invade the intestinal epithelial cells, we selected the duodenum, liver, lung and spleen for pathological analysis. Compared with the control, the NC8-pSIP409-pgsA-VP6-Dcpep group had intact intestinal villi, clear liver structure, clear alveolar margin in the lung, clear spleen red pulp and white pulp, and no pathological changes (Fig 9). The pathological changes in PBS administered mice group were vivid such as congestion of intestinal wall, edema, vacuolar degeneration and integrity destruction of enteric epithelium, compared to the NC8-pSIP409pgsA-VP6-Dcpep and NC8-pSIP409-pgsA groups. In the NC8-pSIP409-pgsA group the border of villi was still clear, but was less vacuolar degeneration and edema of intestinal wall occurred compared to NC8pSIP409-pgsA-VP6-Dcpep which had intact intestinal villi.

\section{Discussion}

The main sources of acute diarrhea in piglets are porcine rotaviruses, and infection with these viruses can lead to mild to severe diarrhea with high mortality and morbidity rates. Porcine rotavirus infection has been an economic concern worldwide among pig breeders. The major prophylactic method for the prevention and control of porcine rotavirus infections is vaccination. There are a number of advantages offered by mucosal immunization that are not offered by other routes of antigen delivery, such as the ability to stimulate both the systemic and local immune responses, cost effectiveness and the ease of administration [21-23].

Specific antibodies are particularly important for humoral immunity therefore specific IgA anti-VP6 protein levels were observed to induce mucosal immune responses from different mucosal surfaces. The oral administration of recombinant L. plantaram NC8-pSIP409-pgsA-VP6-Dcpep stimulated both mucosal slgA and systemic IgG immune responses. Compared to the control group the NC8-pSIP409-pgsA-VP6Dcpep-immunized mice the expression level of specific $\lg G$ in serum and $\operatorname{slg} A$ in intestinal lavage fluid 
were significantly higher than those in empty vector group the specific slgA for VP6 could be isolated from the gastrointestinal tract or faeces. This study recommended protecting pigs from porcine rotavirus infections using vaccination with recombinant $L$. plantaram NC8-pSIP409-pgsA-VP6-Dcpep because this recombinant effectively provoked a significant and specific anti-VP6 IgA response.

The serum from mice immunized with L. plantaram NC8-pSIP409-pgsA-VP6-Dcpep had higher titers of anti-VP6 IgG than the control group. L. plantaram NC8-pSIP409-pgsA-VP6-Dcpep even stimulated elevated IgA levels specific for VP6 compared to the serum from mice immunized with the control NC8pSIP409-pgsA because of the Dcpep mucosal adjuvant. The use of the specific mucosal adjuvanticity of Dcpep was safe, and to stimulate specific anti-porcine rotavirus immunity, an efficient mucosal adjuvant can be used in combination with VP6. When activated in vivo, DCs will stimulate T cells to differentiate into helper T cells, such as Th1 and Th2, which play an important role in secreting IFN-gamma and IL-4 cytokines, respectively. NC8-pSIP409-pgsA-VP6-Dcpep stimulated T cells to secrete more IFN-gamma and IL-4, which resulted in T cells differentiating into Th1 and Th2. This result was consistent with the previous results that Pereira et al. [24].

On the other hand, we also evaluated the humoral immune response, namely the activation of B cells and the expression level of intracellular IgA antibodies. The results confirmed a significant increase in the percentage of IgA+B220+ B cells, indicating a certain immunoregulatory function on the host immune system. The mice orally immunized with NC8-pSIP409-pgsA-VP6-Dcpep demonstrated a virus challengeinduced mean fever peak of $38.6^{\circ} \mathrm{C}$ in the NC8-pSIP409-pgsA-VP6-Dcpep group and $41.1^{\circ} \mathrm{C}$ in the NC8pSIP409-pgsA group 4 to 6 days after the challenge, and 7 to 9 days following challenge, the surviving mice recovered gradually from the clinical signs of PRV infection. Compared with the control, the NC8pSIP409-pgsA-VP6-Dcpep group had intact intestinal villi, clear liver structure, clear alveolar margin in the lung, clear spleen red pulp and white pulp, and no pathological changes.

\section{Conclusion}

The VP6 genes of the porcine rotavirus (PRV) Chinese PRV isolate DN30209 strain expressed by $L$. plantarum NC8 can stimulate antibodies in mice that may be used for the development of vaccines against rotavirus-induced diarrhea in piglets. Rotavirus genes of other serotypes can also be expressed by the L. plantarum NC8 expression system for vaccines or other purposes.

\section{Methods}

\section{Chemicals and enzymes}

All chemicals for the enzyme assay; the cloning, expression, and purification of recombinants; all restriction enzymes such as (Hind III and Xba I), DNA polymerase, RNase-A and proteinase were purchased from Beijing Kang Century Biotechnology Co., Ltd and media components; kits; reagents/adjuvants; and proteins were purchased from Beijing Soleboard Technology Co., Ltd. and were 
provided by the Laboratory of Jilin Provincial Engineering Research Center of Animal Probiotics, in the Jilin Agricultural University of China.

\section{Strains and culture conditions}

\section{L. plantarum NC8 was kindly provided by the Laboratory of Jilin Provincial Engineering Research Center} of Animal Probiotics. L. plantarum was grown anaerobically in MRS broth at $37^{\circ} \mathrm{C}$ without shaking; erythromycin (EM) was added when appropriate to the culture medium at a $10 \mu \mathrm{g} / \mathrm{ml}$ final concentration, and growth was monitored for $12 \mathrm{~h}$. Then, single colonies were grown in MRS liquid medium according to the same procedure as described by $[25,26]$. In the case of interventions with $L$. plantarum, cells were harvested by centrifugation and washed three times with a physiological salt solution (buffer solutions). Porcine rotavirus isolate DN30209 VP6 gene, complete cds (GenBank: JN977137.1) strains were purchased from HaiGene China Co. and provided by the Laboratory of Jilin Provincial Engineering Research Center of Animal Probiotics at the Jilin Agricultural University of China. The expression vector pSIP409-pgsA was obtained from previous work [27] in which it was used for the heterologous expression of target genes (VP6 and NC8) in a prokaryotic expression system.

\section{Mice}

In this model, we used Rotavirus antibody-free adult female Balb/C mice that were 7 weeks of age (weighing 25-30 g) obtained from Beijing Huafukang Biotechnology Co., Ltd. China; these mice are used to investigate the immune responses to the recombinant NC8-pSIP409-pgsA-VP6-Dcpep strain. Forty-five (45) mice were randomly divided into three groups and were housed under standard conditions with free access to food and water. This study was carried out in agreement with the principles established by Jilin Agriculture University Changchun China and guide for the use of laboratory and care animals and all experimental protocols were approved by a Jilin Agriculture University (No. JLAU08201007). The mice were euthanized by using cervical dislocation method after finishing the experiment.

\section{Mouse anti-VP6 antibodies}

The detection of the surface fluorescence intensity of NC8-pSIP409-pgsA-VP6-Dcpep by flow cytometry was performed as follows: NC8-pSIP409 and NC8-pSIP409-pgsA-VP6-Dcpep were induced in overnight culture. The bacterial solution was centrifuged for 1 minute at $12000 \mathrm{rpm}$ and then precipitated and washed three times with PBS containing 0.5\% BSA. The sediment was collected and rabbit anti-VP6 serum (1:2000 dilutions) was added and mixed well. Then the solution was incubated on a $60 \mathrm{rpm}$ shaking bed at room temperature for 1 hour. After washing three times with PBS containing $0.2 \%$ Tween 20 , the sediment was collected and FITC-labeled goat anti-rabbit IgG fluorescent antibody (1:100 dilution) was added; the solution was incubated at room temperature for 1 hour at $60 \mathrm{rpm}$. After 3 more washes with PBS containing $0.2 \%$ Tween 20,300 L PBS was added, and the solution was moved into the flow 
tube after passing through the membrane. Flow cytometry was used to detect the results. The antiporcine rotavirus VP6 (anti-VP6) serogroup C antibody used in the immunofluorescence and Western blot analyses was purchased from Arigo Biolaboratories.

\section{Expression plasmid construction}

\section{Polymerase chain reaction (PCR) of VP6 gene proteins}

A gene fragment obtained from the genome of PRV strain DN30209 encoding the main structural polypeptide of VP6 that was 1356 bp in length was amplified by polymerase chain reaction (PCR) (porcine rotavirus isolate DN30209 VP6 gene, complete cds GenBank: JN977137.1). Specific primers were designed with Primers Premier 5.0 to amplify the full length of the $48 \mathrm{kDa}$ VP6 protein gene based on the sequence data given for the VP6 gene fragment, which was $1356 \mathrm{bp}$ of the porcine rotavirus genome. The restriction sites were as follows: 5' GGATCCCAAGACAACACCTGGTATG 3' (forward) containing a Hind III site and CTCGAG 3'GCTGACACCTTCGACTTTC 5' (reverse) containing an Xba I site. The PCR was carried out in a standard $50 \mathrm{ml}$ reaction for the amplification of the VP6 gene using the primer sets following standard protocols. The tubes were then placed in a thermocycler, and the PCR conditions for amplification were as follows: predenaturation at $94^{\circ} \mathrm{C}$ for 5 minutes, denaturation at $94^{\circ} \mathrm{C}$ for 30 seconds, annealing at $55^{\circ} \mathrm{C}$ for 30 seconds, extension at $72^{\circ} \mathrm{C}$ for 30 cycles and extension at $72^{\circ} \mathrm{C}$ for 10 minutes. After the reaction was finished, the 2 L PCR product was electrophoresed on a $0.8 \%$ agarose gel.

Electroporation of L. plantarum was carried out as follows: $10 \mu \mathrm{l}$ of plasmid was gently added to $100 \mu \mathrm{l}$ of $L$. plantarum NC8, mixed gently for $5 \mathrm{~min}$ at $4^{\circ} \mathrm{C}$ and subjected to an electric pulse. The mixture of plasmid DNA and L. plantarum NC8 was then anaerobically incubated without EM in MRS medium for 2 $\mathrm{h}$ at $37^{\circ} \mathrm{C}$. Recombinant NC8-pSIP409-pgsA-VP6-Dcpep was selected on MRS agar medium including EM. The transformants with L. plantarum NC8 sequences were verified by plasmid DNA sequencing.

\section{Flow cytometry}

The cells were carefully washed with PBS, centrifuged at $12000 \mathrm{rpm}$ for $5 \mathrm{~min}$ at $4^{\circ} \mathrm{C}$, and washed twice with PBS; then, $10 \mu \mathrm{L}$ of cells were added to each flow cytometry tube and incubated at room temperature for $20 \mathrm{~min}$ in the dark. The cells were washed twice with cold PBS, centrifuged at $4^{\circ} \mathrm{C}$ and $2000 \mathrm{rpm}$ for 5 min; the cell suspension was centrifuged, and approximately $300 \mu \mathrm{L}$ was transferred to a flow tube. The effect of lactic acid bacteria on the activation B cells was detected by flow cytometry. The data were analyzed using FlowJo 7.6.1 software [28]. 


\section{Protein expression and sodium dodecyl sulfate polyacrylamide gel electrophoresis, Western blot analysis}

NC8-pSIP409 and NC8-pSIP409-pgsA-VP6-Dcpep frozen at - 80 $\mathrm{C}$ were subcultured. The second generation of bacterial liquid was added to $50 \mathrm{~mL}$ of MRS liquid medium (Em+) at a 1:100 ratio. Anaerobic culture was carried out in a $30^{\circ} \mathrm{C}$ incubator until the OD value was 0.3 . SppIP was added at a ratio of 1:400 for overnight induction. The overnight culture solution was centrifuged for 10 minutes at $4^{\circ} \mathrm{C}$ and $5000 \mathrm{~g}$, and the supernatant was discarded. The supernatant was washed three times with cold PBS. The precipitation was suspended in $500 \mu \mathrm{L}$ of pyrolysis buffer. The supernatant was digested at $120 \mathrm{rpm}$ for 30 minutes in a shaker at $37^{\circ} \mathrm{C}$ and centrifuged at $10000 \mathrm{~g}$ for 30 minutes. When the supernatant was collected, the soluble cell wall component, VP6, was contained in the supernatant. A total of $160 \mu \mathrm{L}$ was removed from the supernatant and added to $40 \mu \mathrm{L}$ of the $5 \times$ SDS buffer. The protein was denatured by boiling at $100^{\circ} \mathrm{C}$ for 5 minutes. The supernatant was centrifuged for 2 min at 12000 rpm. SDS-PAGE was performed.

\section{SDS-PAGE}

Two plates of glue were allocated for SDS-PAGE, one of which was dyed and decolorized according to the above method and observed on the machine; the other was carried out according to the following method.

\section{Transmembrane}

After the SDS-PAGE was finished, the gel was removed, and transfer filter paper was soaked in transfer buffer for $30 \mathrm{~min}$. The PVDF membrane was first activated for $30 \mathrm{~s}$ with methanol and then soaked in the transfer buffer. According to the size of the gel, the filter paper and PVDF film were cut and then placed in the transfer buffer. The order was sponge, 3 layers of filter paper, PVDF film, gel, 3 layers of filter paper and sponge. The bubbles generated between each layer were removed by glass rods and then the gel was placed into the transfer clamp. The gel side was connected with the negative electrode. The PVDF film was connected with the positive pole at a constant current of $200 \mathrm{~mA}$ and transference occurred on ice for approximately 1 hour.

\section{Immunofluorescence analysis}

To examine the surface expression of VP6 protein in NC8-pSIP409-pgsA-VP6-Dcpep, we used immunofluorescence as follows: Briefly, $1 \mathrm{~mL}$ of the SPPIP-induced cultures were harvested when they reached an OD of 600 and then resuspended in $1 \mathrm{ml}$ of sterile PBS 3\%; then, goat serum albumin with anti-VP6 antibodies was added and incubated at $-4^{\circ} \mathrm{C}$ overnight. Then, the cells were pelleted and washed three times with sterile PBS 3\% Tween 20. Next, the cell antibody mixtures were incubated at room 
temperature in the dark with fluoreoscein isothiocyanate (FITC)-conjugated mouse anti-porcine IgG containing 1\% Evans blue. We washed the cells three times with PBS 3\% Tween 20 and then transferred them to a glass slide for air drying. Noninduced recombinant NC8-pSIP409-pgsA was used as a negative control. Confocal microscopy was used to perform the analysis.

\section{Immunizations}

The induced culture of NC8-pSIP409-pgsA-VP6-Dcpep was centrifuged as described above. Then, we washed the cell pellets once with sterile PBS and resuspended them in $1 \mathrm{ml}$ of PBS pH 7.4. Fifteen Balb/C mice from each group were orally administered $0.2 \mathrm{ml}$ of $10^{9}$ colony forming units $/ \mathrm{ml}$ (c.f.u. $/ \mathrm{ml}$ ) of the recombinant NC8-pSIP409-pgsA-VP6-Dcpep, NC8-pSIP409-pgsA and normal saline. All groups of mice were immunized nine times, that is, on days 0,1 and 2, and they received boosters on days 14,15 and 16 and again on days 28,29 and 30 .

\section{Enzyme-linked immunosorbent assay (ELISA)}

To examine specific anti-VP6 antibodies, mouse serum was collected on days 14, 35 and 42 after immunization and centrifuged at $4000 \mathrm{rpm}$ for $15 \mathrm{~min}$ at $4^{\circ} \mathrm{C}$ to obtain the serum, which was stored at $-80^{\circ} \mathrm{C}$ and then analyzed by ELISA as described previously [29]. ELISA was performed to determine the levels of specific IgG and slgA antibodies [30]. Feces samples were also collected at 14, 35 and 42 days after immunization. The serum samples (1:100) and fecal samples (1:10) were added separately after dilution and, following incubation for $2 \mathrm{~h}$ at $37^{\circ} \mathrm{C}$, the wells were rinsed 5 times. Then, goat anti-mouse IgA-Biotin and goat anti-mouse IgG $(\mathrm{H}+\mathrm{L})$-Biotin (Southern Biotechnology, Birmingham, AL) were added separately, and the plate was incubated at $37^{\circ} \mathrm{C}$ for one hour, after which streptavidin-HRP (Southern Biotechnology, Birmingham, AL) secondary antibody was added and the plate was incubated at $37^{\circ} \mathrm{C}$ for 10 min. Finally, chromogen solution $A$ and $B$ and stop solution were added, after which the color intensity was measured at $450 \mathrm{~nm}$. The end-point titers $\left(\log _{2}\right)$ were defined as the highest dilution yielding an absorbance that was two and three times higher than the background for the serum and fecal samples, respectively [31].

\section{Detection of cytokines}

The serum samples were examined for specific cytokines, including IFN- $\gamma$ and IL-4, using an ELISA kit (LIUHEBIO, Wuhan, China) according to the manufacturer's instructions.

\section{Response of Vaccinated Mice to PRV Challenge}

A virus challenge experiment was performed to evaluate the protective effect of NC8-pSIP409-pgsA-VP6DCpep against PRV infection in mice post vaccination. Three weeks after the last immunization, the 
immunized mice were challenged (by oral gavage) with $100 \mathrm{ID}_{50}$ of a Chinese porcine rotavirus isolate, strain DN30209,3 weeks after the final immunization.

\section{Statistical analysis}

Flowjo 7.6.1 software was used for flow cytometry analysis, GraphPad Prism 5 software was used for mapping and statistical data, and one-way ANOVA was used for statistical analysis of the differences between groups $(*, P<0.05 ; * \star, P<0.01 ; * \star *, P<0.001)$.

\section{Abbreviations And Acronynys}

$\mathrm{BALB} / \mathrm{C} \quad$ albino laboratory bred strain of house mouse

CD Cluster of differentiation

DCpep Dendritic cell targeting peptide

ELISA Enzyme-linked immuno sorbent assay

IFN interferon

$\lg \mathrm{A} \quad$ immunogloblin $\mathrm{A}$

Ig $\quad$ immunogloblin $\mathrm{G}$

IL $\quad$ interleukin

IFN- $\quad$ interferon gamma

L. Lactobacillus

MLN Mesenteric lymph nodes

PP Peyer's patch

PBS Phosphate buffered saline

slgA immunoglobulin $\mathrm{A}$

\section{Declarations}

Ethics approval and consent to participate:

This study was carried out in agreement with the principles established by Jilin Agriculture University Changchun China and guide for the use of laboratory and care animals and all experimental 
protocols were approved by a Jilin Agriculture University (No. JLAU08201007).

Consent for publication: Not applicable

Availability of data and materials: will be provided after acceptance

\section{Competing Interests:}

Author1 declares that she has no conflict of interest.

Author 2 declares that he has no conflict of interest.

Author 3 declares that she has no conflict of interest.

Author 4 declares that he has no conflict of interest

Funding: This work was supported by The National Key Research and Development Program of China (2017YFD0501000). The funder had no role in study design, preparation of the manuscript, data collection, decision to publish and analysis.

Authors' contributions Author 1 SMS -searching data, wrote manuscript and acted as corresponding author

Author 2 WY -editing manuscript

Author $3 \mathrm{GY}$-editing the manuscript and supervision of the manuscript

Author $4 \mathrm{CW}$ - editing the manuscript and supervision of the manuscript

\section{All authors have read and approved the manuscript}

\section{Acknowledgement:}

We are thankful to the Jilin Agricultural University for the internet to download Data for this study.

\section{References}

1. Kava R: Rotavirus Vaccines Do More Than Prevent Diarrhea. American Council on Science and Health 2018.

2. Hull JJ, Marthaler D, Rossow S, Ng TFF, Montmayeur AM, Magana L, Moon S-S, Jiang B: Genomic Sequence of the First Porcine Rotavirus Group H Strain in the United States. Genome Announcements 2016, 4(2):e01763-01715.

3. Marthaler D, Rossow K, Gramer M, Collins J, Goyal S, Tsunemitsu H, Kuga K, Suzuki T, Ciarlet M, Matthijnssens J: Detection of substantial porcine group B rotavirus genetic diversity in the United States, resulting in a modified classification proposal for G genotypes. Virology 2012, 433(1):85-96. 
4. Matthijnssens J, Ciarlet M, McDonald SM, Attoui H, Banyai K, Brister JR, Buesa J, Esona MD, Estes MK, Gentsch JR et al: Uniformity of rotavirus strain nomenclature proposed by the Rotavirus Classification Working Group (RCWG). Archives of virology 2011, 156(8):1397-1413.

5. Paul PS, Lyoo YS: Immunogens of rotaviruses. Veterinary Microbiology 1993, 37(3):299-317.

6. Gachanja E, Buza J, Petrucka P: Prevalence of Group A Rotavirus in Piglets in a Peri-Urban Setting of Arusha, Tanzania. Journal of Biosciences and Medicines 2016, 04(01):37-44.

7. Wilhelm B, Waddell L, Greig J, Rajic A, Houde A, McEwen SA: A scoping review of the evidence for public health risks of three emerging potentially zoonotic viruses: hepatitis $E$ virus, norovirus, and rotavirus. Preventive veterinary medicine 2015, 119(1-2):61-79.

8. Nataraju SM, Chattopadhyay UK, Krishnan T: A study on the possibility of zoonotic infection in rotaviral diarrhoea among calves and buffalo calves in and around Kolkata, India. European review for medical and pharmacological sciences 2009, 13(1):7-11.

9. Ambily R, Mini M, John K, Nair GK: First report of bovine rotavirus from Kerala. Biosciences, Biotechnology Research Asia 2009, 6(1):245-249.

10. Manuja BK, Prasad M, Manuja A, Gulati BR, Prasad G: A novel genomic constellation (G10P[3]) of group A rotavirus detected from buffalo calves in northern India. Virus research 2008, 138(1-2):36-42.

11. Ghosh S, Samajdar S, Sinha M, Kobayashi N, Taniguchi K, Naik TN: Molecular characterization of rare bovine group A rotavirus G15P[11] and G15P[21] strains from eastern India: identification of simian SA11-like VP6 genes in G15P[21] strains. Virus genes 2008, 37(2):241-249.

12. Vlasova AN, Amimo JO, Saif LJ: Porcine Rotaviruses: Epidemiology, Immune Responses and Control Strategies. 2017, 9(3):48.

13. Dewey C, Carman S, Pasma T, Josephson G, McEwen B: Relationship between group A porcine rotavirus and management practices in swine herds in Ontario. The Canadian Veterinary Journal 2003, 44(8):649-653.

14. Shaw AL, Rothnagel R, Zeng CQY, Lawton JA, Ramig RF, Estes MK, Prasad BVV: Rotavirus structure: interactions between the structural proteins. In: Viral Gastroenteritis. edn. Edited by Chiba S, Estes MK, Nakata S, Calisher CH. Vienna: Springer Vienna; 1996: 21-27.

15. Chitambar SD, Arora R, Kolpe AB, Yadav MM, Raut CG: Molecular characterization of unusual bovine group A rotavirus G8P[14] strains identified in western India: Emergence of P[14] genotype. Veterinary Microbiology 2011, 148(2):384-388.

16. Malik YS, Sharma K, Vaid N, Chakravarti S, Chandrashekar KM, Basera SS, Singh R, Minakshi, Prasad G, Gulati BR et al: Frequency of group A rotavirus with mixed $\mathrm{G}$ and $\mathrm{P}$ genotypes in bovines: predominance of G3 genotype and its emergence in combination with G8/G10 types. Journal of Veterinary Science 2012, 13(3):271-278.

17. Matson DO, O'Ryan ML, Herrera I, Pickering LK, Estes MK: Fecal antibody responses to symptomatic and asymptomatic rotavirus infections. The Journal of infectious diseases 1993, 167(3):577-583.

18. Coulson BS, Grimwood K, Hudson IL, Barnes GL, Bishop RF: Role of coproantibody in clinical protection of children during reinfection with rotavirus. Journal of clinical microbiology 1992 , 
30(7):1678-1684.

19. Pavan S, Hols P, Delcour J, Geoffroy MC, Grangette C, Kleerebezem M, Mercenier A: Adaptation of the nisin-controlled expression system in Lactobacillus plantarum: a tool to study in vivo biological effects. Applied and environmental microbiology 2000, 66(10):4427-4432.

20. Curiel TJ, Morris C, Brumlik M, Landry SJ, Finstad K, Nelson A, Joshi V, Hawkins C, Alarez X, Lackner A et al: Peptides identified through phage display direct immunogenic antigen to dendritic cells. Journal of immunology (Baltimore, Md : 1950) 2004, 172(12):7425-7431.

21. Azegami T, Yuki Y, Kiyono H: Challenges in mucosal vaccines for the control of infectious diseases. International immunology 2014, 26(9):517-528.

22. Kim S-H, Jang Y-S: The development of mucosal vaccines for both mucosal and systemic immune induction and the roles played by adjuvants. Clin Exp Vaccine Res 2017, 6(1):15-21.

23. Patel $H$, Yewale $C$, Rathi MN, Misra A: Mucosal immunization: a review of strategies and challenges. Critical reviews in therapeutic drug carrier systems 2014, 31(4):273-303.

24. Pereira VB, da Cunha VP, Preisser TM, Souza BM, Turk MZ, De Castro CP, Azevedo MSP, Miyoshi A: Lactococcus lactis carrying a DNA vaccine coding for the ESAT-6 antigen increases IL-17 cytokine secretion and boosts the BCG vaccine immune response. Journal of applied microbiology 2017, 122(6):1657-1662.

25. Yang WT, Yang GL, Shi SH, Liu YY, Huang HB, Jiang YL, Wang JZ, Shi CW, Jing YB, Wang CF: Protection of chickens against H9N2 avian influenza virus challenge with recombinant Lactobacillus plantarum expressing conserved antigens. Appl Microbiol Biotechnol2017, 101(11):4593-4603.

26. Yang G, Jiang Y, Yang W, Du F, Yao Y, Shi C, Wang C: Effective treatment of hypertension by recombinant Lactobacillus plantarum expressing angiotensin converting enzyme inhibitory peptide. Microbial Cell Factories 2015, 14(1):202.

27. Huang KY, Yang GL, Jin YB, Liu J, Chen HL, Wang PB, Jiang YL, Shi CW, Huang HB, Wang JZ et al: Construction and immunogenicity analysis of Lactobacillus plantarum expressing a porcine epidemic diarrhea virus S gene fused to a DC-targeting peptide. Virus research 2018, 247:84-93.

28. Kikuchi Y, Kunitoh-Asari A, Hayakawa K, Imai S, Kasuya K, Abe K, Adachi Y, Fukudome S, Takahashi Y, Hachimura S: Oral administration of Lactobacillus plantarum strain AYA enhances IgA secretion and provides survival protection against influenza virus infection in mice. PLoS One 2014, 9(1):e86416.

29. Wang Z, Yu Q, Gao J, Yang Q: Mucosal and systemic immune responses induced by recombinant Lactobacillus spp. expressing the hemagglutinin of the avian influenza virus H5N1. Clinical and vaccine immunology : CVI2012, 19(2):174-179.

30. Yang WT, Yang GL, Wang Q, Huang HB, Jiang YL, Shi CW, Wang JZ, Huang KY, Jin YB, Wang CF: Protective efficacy of Fc targeting conserved influenza virus M2e antigen expressed by Lactobacillus plantarum. Antiviral Res 2017, 138:9-21.

31. Shi SH, Yang WT, Yang GL, Zhang XK, Liu YY, Zhang LJ, Ye LP, Hu JT, Xing X, Qi C et al: Lactobacillus plantarum vaccine vector expressing hemagglutinin provides protection against H9N2 challenge infection. Virus research 2016, 211:46-57. 


\section{Figures}

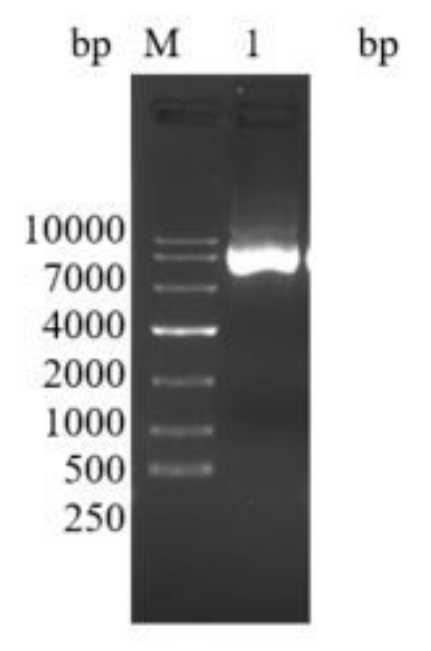

pSIP409-pgsA-VP6-

Dcpep plasmid

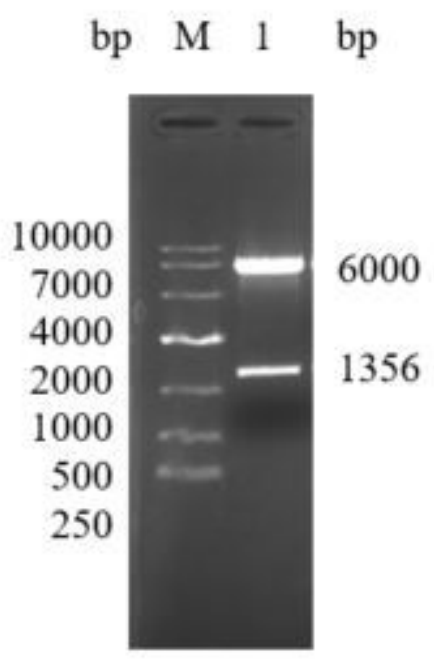

Recombinant pSIP409-pgsAVP6-Dcpep plasmid analysis by restriction enzyme digestion.

Sequence analysis confirmed that pSIP409-pgsA-VP6-Dcpep clone contained a full-length copy of gene.

\section{Figure 1}

Agarose Gel extraction of VP6 gene segment and vector 
NC8-pSIP409-pgsA-VP6-DCpep
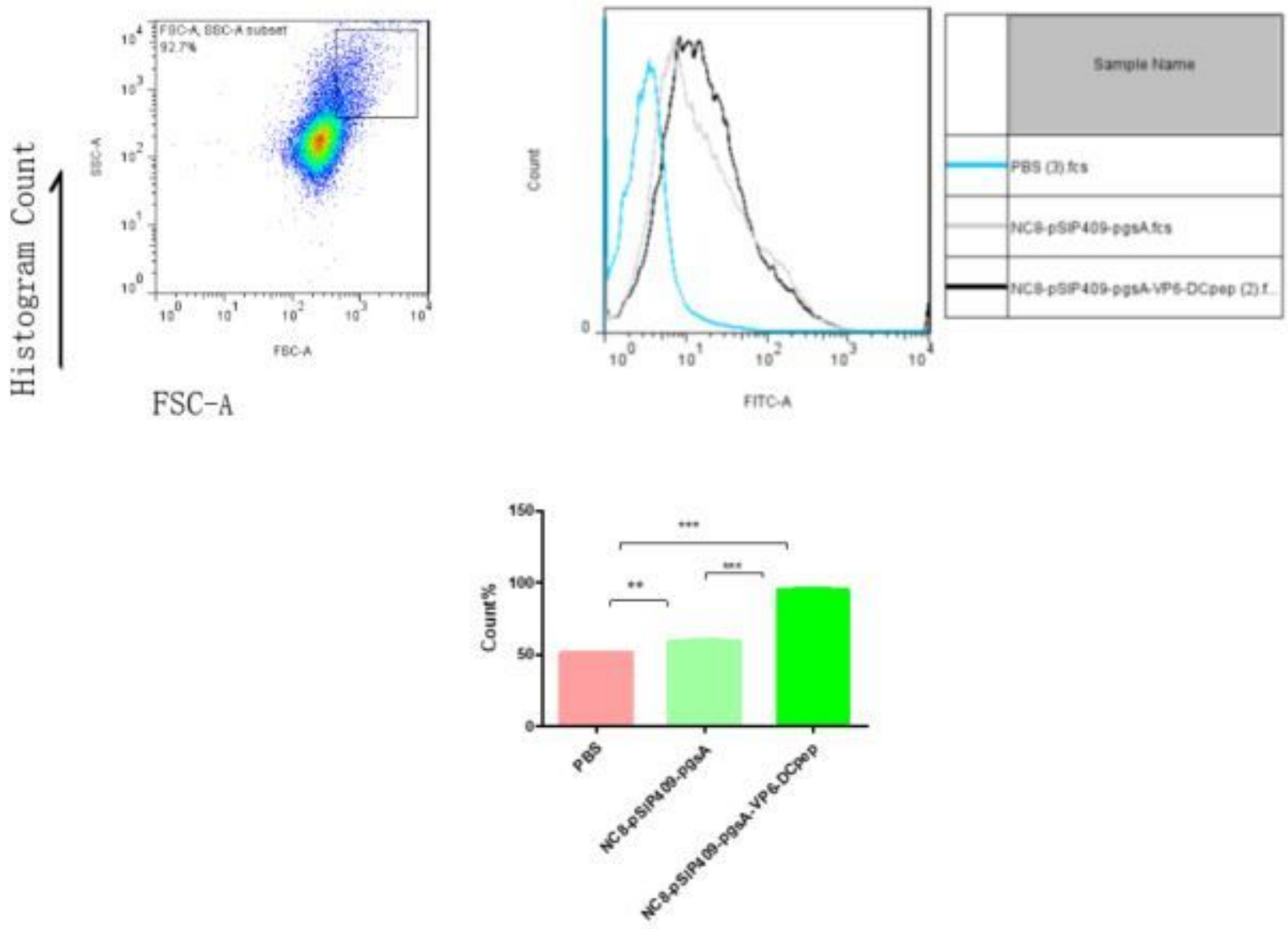

Figure 2

Flow cytometry analysis of VP6 surface expression in L. plantaram NC8 


\section{SDS-PAGE}

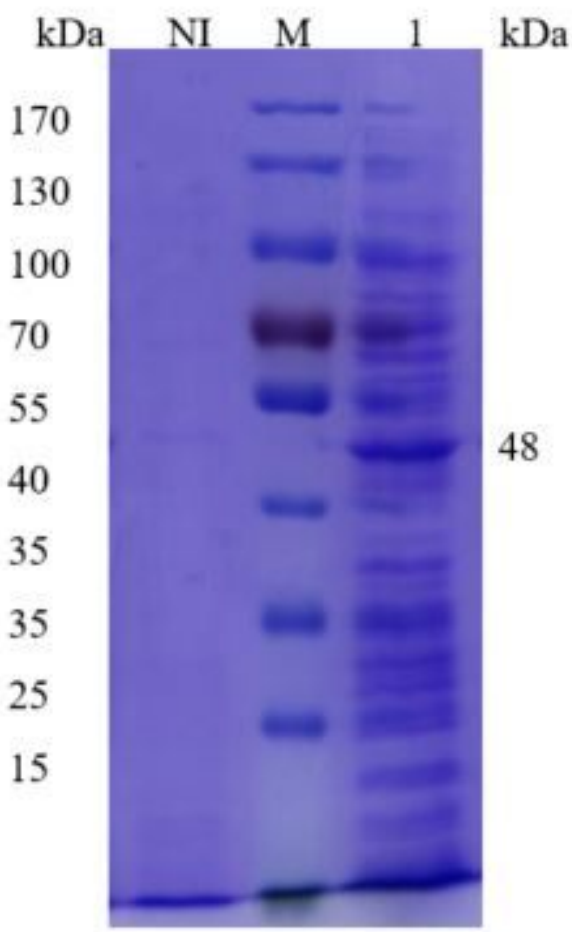

Expression analysis of NC8pSIP409-pgsA-VP6-Dcpep Coomasie-blue-stained SDS-PAGE of $L$. plantaram NC8.10 $\mu \mathrm{l}$ was loaded on $10 \%$ SDS-PAGE gel at $150 \mathrm{~V}$. Lane NI= uninduced NC8pSIP409-pgsA, Lane $\mathrm{M}=$ Marker, Lane 1-3 = induced NC8-pSIP409pgsA-VP6-Dcpep
Western blot

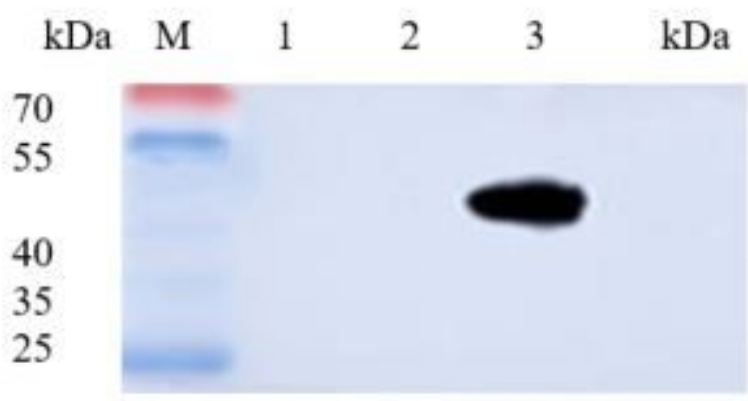

Expression analysis of recombinant NC8-pSIP409-pgsA-VP6-Dcpep / by western blot.

Lane $\mathrm{M}=$ low molecular weight protein marker Lane 1-2 = Uninduced NC8pSIP409-pgsA

Lane 3 = induced NC8-pSIP409pgsA-VP6-Dcpep

\section{Figure 3}

Expression analysis of NC8-pSIP409-pgsA-VP6-Dcpep by SDS-PAGE and Western blot 
PBS

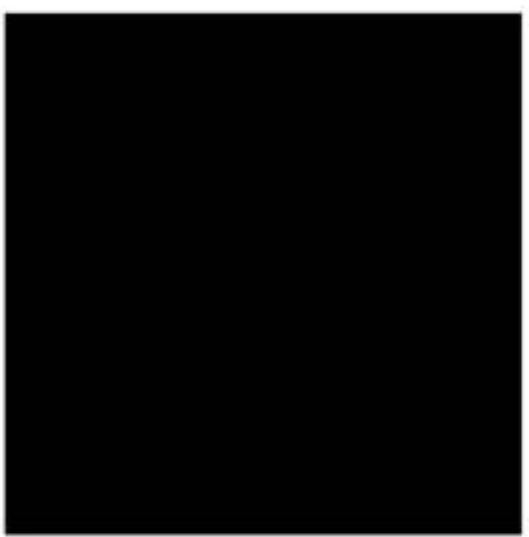

NC8-pSIP409-pgsA

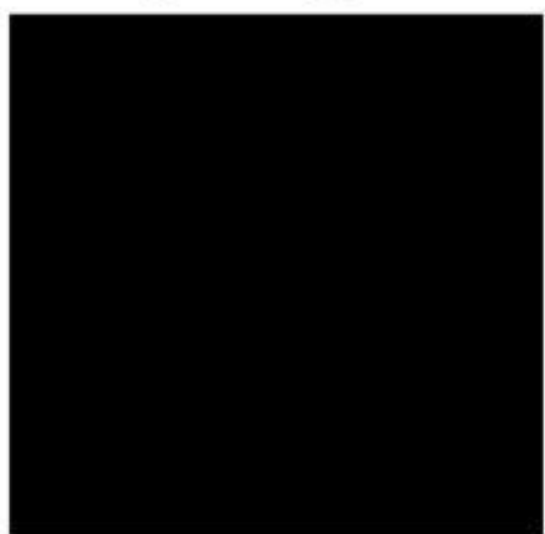

NC8-pSIP409-pgsA-VP6-Dcpep

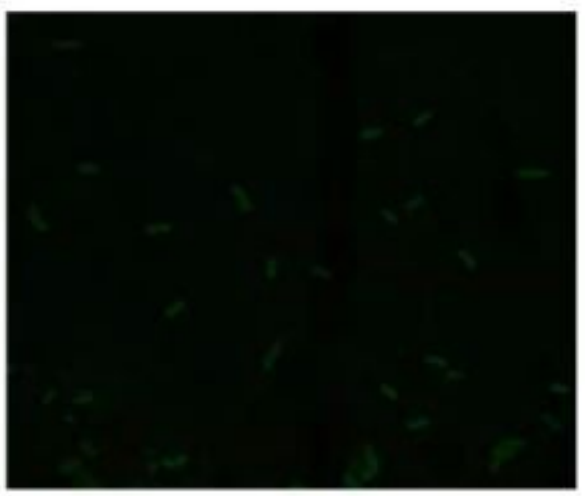

Figure 4

immunofluorescence assay analysis of VP6 surface expression in L. plantaram NC8
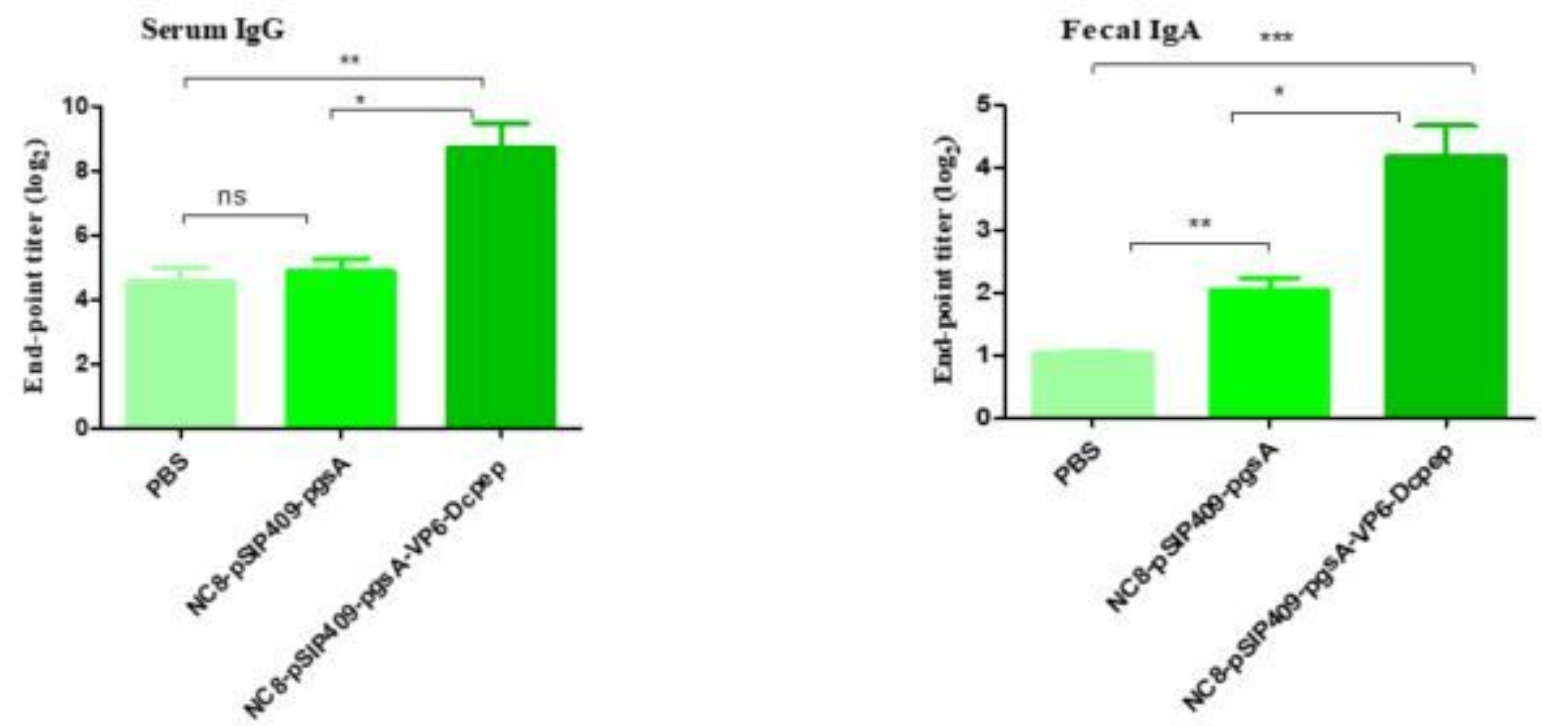

Figure 5

(A) Changes of specific IgG content in serum and (B) Changes of specific slgA content in fecal 
Serum II-4

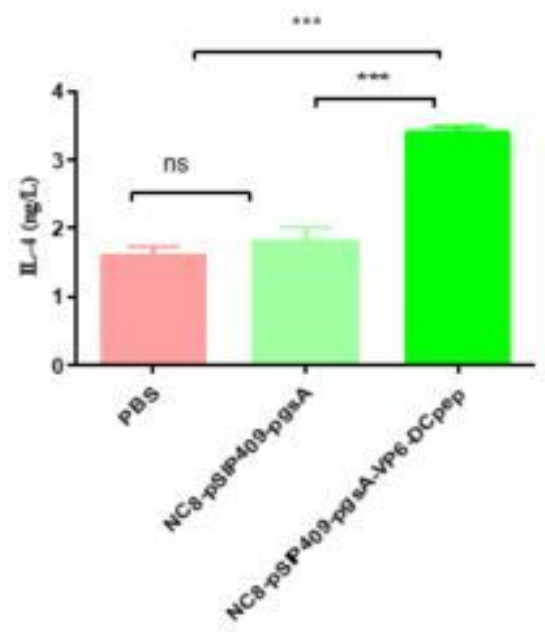

Serum IFN- $\gamma$

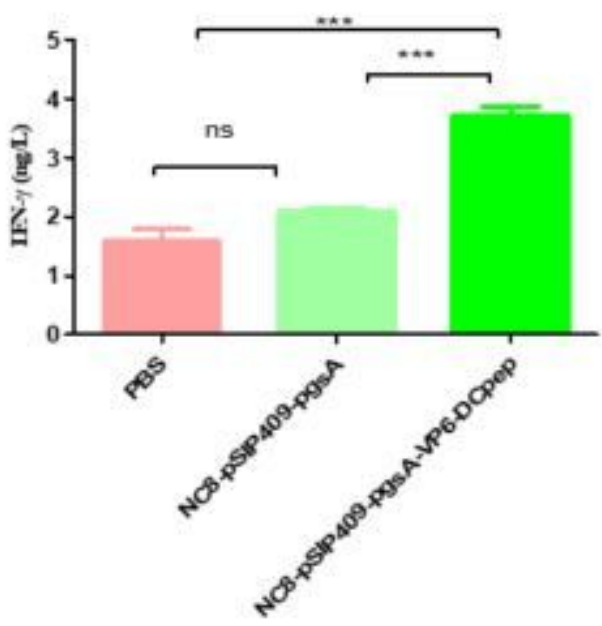

Figure 6

the contents of two cytokines in serum were determined by ELISA 
PBS

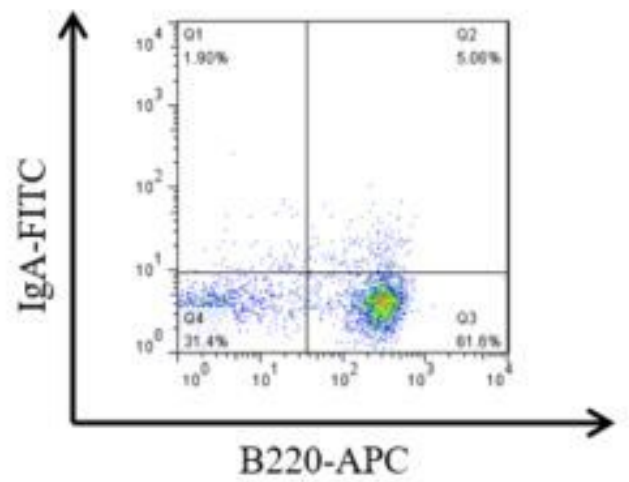

NC8-pSIP409-pgsA

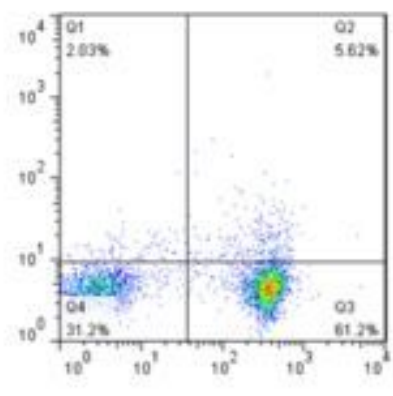

NC8-pSIP409-pgsA-VP6-Dcpep

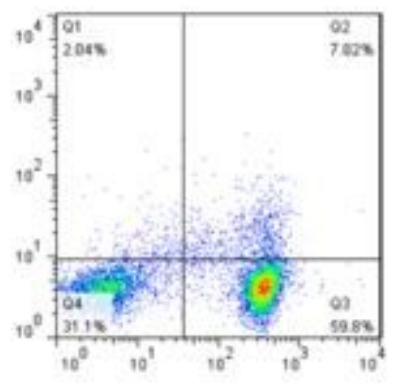

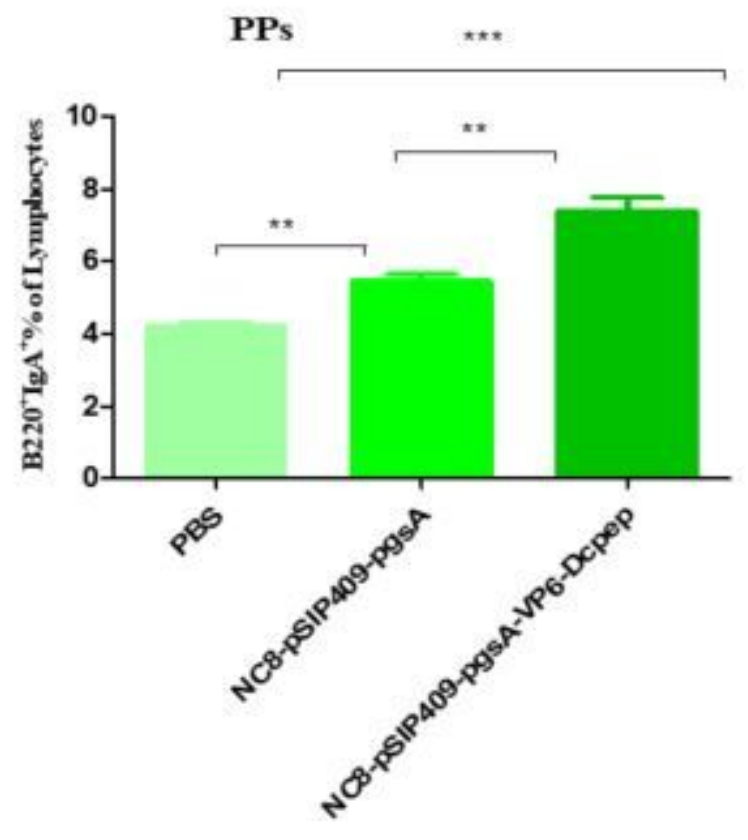

Figure 7

the expression of $B 220+\lg A+B$ cells in mouse PPs 
A

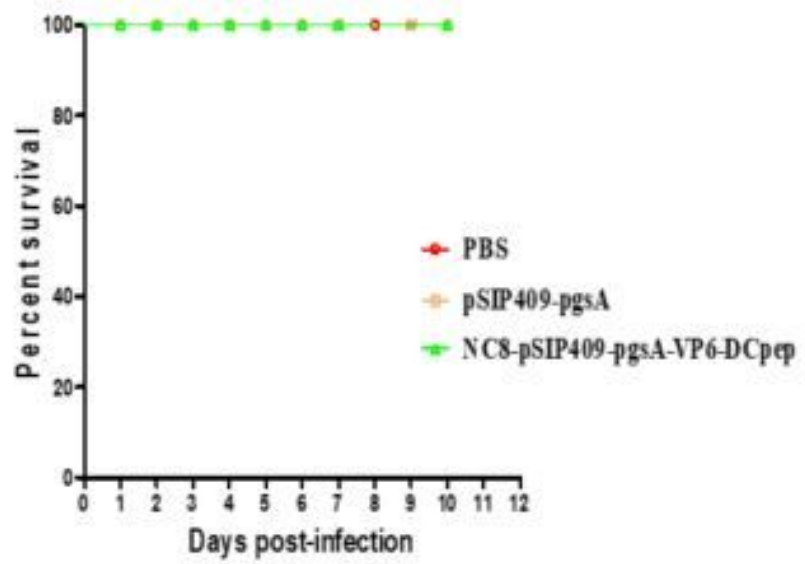

B

$\rightarrow$ PBS

$=$ NC8-pSIP409-pgsA

- NC8-pSIP409-pgsA-VP6-DCpep

\section{Figure 8}

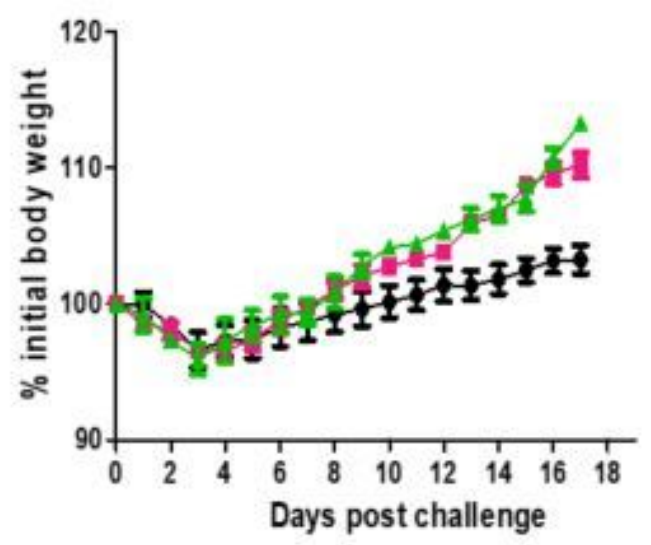

Effects on survival percentages $(A)$ indexes and body weights $(B)$ after immunization

NC8-pSIP409-

PBS
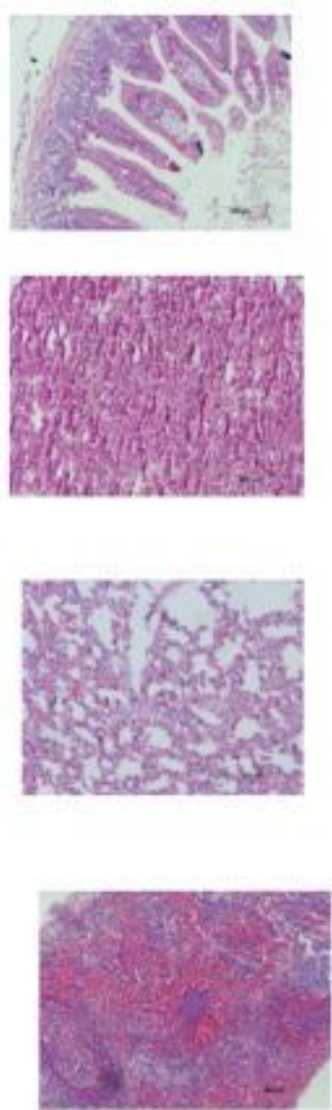

pgsA
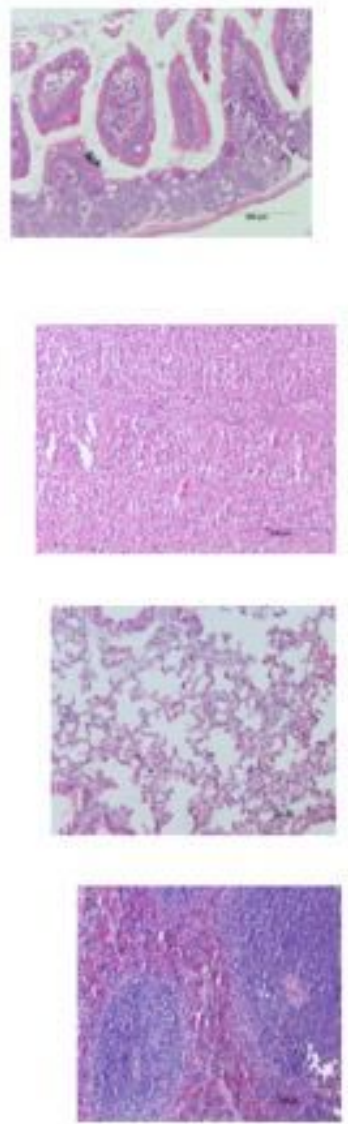

NC8-pSIP409-

pgsA-VP6-

Dcpep

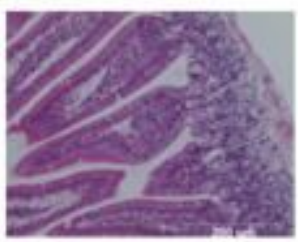

Small intestine

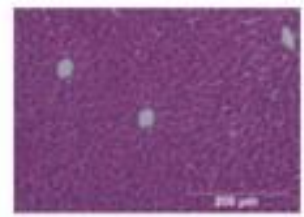

Liver

Lung

Spleen 


\section{Figure 9}

Histopathological changes in mice infected with Chinese porcine rotavirus isolate DN30209 strains following immunization

\section{Supplementary Files}

This is a list of supplementary files associated with this preprint. Click to download.

- CHECKLISTBMC.docx 